\title{
Definición de una metodología optimizada para la simulación del desgaste en materiales metálicos
}

\author{
A. Cruzado*, A. Zabala*, M.A. Urchegui** y X. Gómez*
}

\begin{abstract}
Resumen
La simulación del desgaste mediante el Método de Elementos Finitos (MEF) es una técnica que presenta grandes ventajas para la mejora del diseño de componentes ya que permite reducir el coste que conlleva la experimentación (tiempo de ensayo, materiales, medidas tribológicas). En el caso concreto de la optimización de sistemas multihilo sometidos a fretting, como es el caso de los cables metálicos donde se dan contactos cilindro-cilindro, este método puede resultar especialmente eficiente. Sin embargo, la simulación del desgaste resulta una técnica compleja ya que consta de dos núcleos importantes: por un lado, la resolución del contacto y, por otro, la introducción del modelo de desgaste, requiriendo, para ello, de un coste computacional muy importante. Por ello, en este trabajo se define una metodología a seguir con el fin de optimizar el modelo, en términos de tiempo computacional, para un sistema bloque-cilindro. La metodología definida sería aplicable a todos los modelos de desgaste, de geometrías más complejas. Finalmente, se introduce una técnica que consiste en la introducción de un acelerador de desgaste variable y autoajustable, teniendo en cuenta la evolución de la presión máxima de desgaste y el incremento umbral de desgaste. Esta técnica resulta indispensable en cuerpos sometidos a grandes desgastes, reduciendo el tiempo computacional en un $87 \%$ y un error inferior al $1 \%$.
\end{abstract}

\section{Optimized methodology for the wear simulation of metallic materials}

\begin{abstract}
The wear simulation with finite element method (FEM) presents great advantages for the improvement of components, because of the reduction in the cost in comparison to the experimental procedure (test time, materials, tribological measurements). In the case of wire rope optimization, where one of the great problems is wear due to fretting in cylinder-cylinder contact points, this method could be efficient. Nevertheless, the wear simulation results in a complex methodology: on the one hand is needed to solve the contact problem and on the other hand the introduction of the wear model. For this reason, this methodology results in high computational time consuming. Consequently an efficient methodology for the optimization of the model, in terms of the reduction of computational time, is presented in this work for a block-on-ring system. Finally a variable wear speed factor and auto-fitting with respect to the evolution of maximum contact pressure and with respect to the maximum limit wear increment is introduced. This is necessary, in systems where high amount of wear is presented with a reduction of $87 \%$ in computational time and an error of less than $1 \%$.
\end{abstract}

Keywords Wear; Finite element; Variable speed factor; Metallic materials; Optimized methodology.

\section{INTRODUCCIÓN}

Los cables metálicos son elementos mecánicos formados por un conjunto de hilos o alambres de acero trefilado, trenzados en forma de cordones, los cuales, a su vez, están enrollados alrededor de un núcleo central o alma que puede ser metálica, textil o sintética, como se puede apreciar en la figura 1. Este tipo de construcción les proporciona una gran resistencia a tracción y flexibilidad a flexión por lo que siguen siendo uno de los elementos estructurales mas utilizados en el trasporte vertical (ascensores).

Sin embargo, a lo largo de su vida útil, están sometidos a miles de ciclos de flexión en su paso por las diferentes poleas (de tracción o desvío) y a una determinada fuerza de tracción. Este modo de trabajo implica que los principales modos de fallo observado en este tipo de cables son la rotura de hilos por fatiga

\footnotetext{
* Mondragon Goi Eskola Politeknikoa, Mondragon Unibertsitatea, Loramendi 4, 20500 Arrasate-Mondragon (Spain). E-mail: acruzado@eps.mondragon.edu. TIfno: +34 9437396 92. Fax: +34 943791536

** ORONA eic, Polígono Industrial Lastaola s/n, 20120 Hernani (Spain).
} 


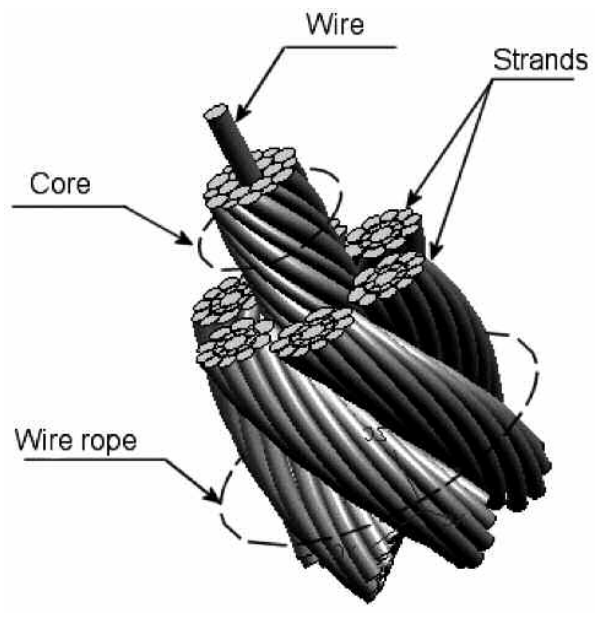

Figura 1. Cable metálico.

\section{Figure 1. Wire rope.}

y la disminución de la sección portante como consecuencia del desgaste por fretting en los puntos de contacto entre hilos ${ }^{[1]}$.

Debido a la complejidad de su construcción y a la dificultad de detectar la evolución del desgaste durante su vida útil, en los puntos de contacto, la aplicación de una herramienta que permita entender su comportamiento ante el desgaste puede resultar muy beneficiosa a la hora de realizar un diseño optimizado de los cables.

En este punto, la simulación del desgaste por MEF (Método de Elementos Finitos) está resultando una técnica muy interesente, a la hora del estudio tensional a medida que los cuerpos se desgastan, ya que la evolución de la geometría y su nuevo estado tensional puede llevar, con mayor exactitud, a la predicción de vida de cualquier componente sometido a desgaste.

La metodología MEF utilizada hasta ahora consiste en un proceso iterativo formado por diferentes etapas $^{[2-8]}$ :

- Cálculo del contacto por medio del MEF.

- Extracción de las presiones y deslizamientos relativos nodales.

- Aplicación de la ley de desgaste, para obtener los desplazamientos nodales para un determinado incremento de deslizamiento.

- Realización, nuevamente, del cálculo del contacto y los pasos precedentes hasta llegar al deslizamiento total requerido.

Por ello, son diferentes autores los que han abordado el tema de simulación en desgaste, tanto en deslizamientos continuos ${ }^{[2-4]}$ como en condiciones de deslizamiento alternativo y fretting ${ }^{[5-7]}$. Todos ellos basan su metodología en modelos de simulación bidimensionales simples, para poder realizar su validación y consiguiente aplicación en modelos de mayor complejidad ${ }^{[3}$ y 8$]$.

En el presente trabajo se define una metodología a seguir a la hora de simular cualquier par tribológico sometido a desgaste, con el fin de obtener resultados óptimos en el menor tiempo posible. Para ello, se utiliza un sistema sencillo bloque-cilindro (block-onring) con el fin de analizar su validez. De este modo, se desarrolla y optimiza la metodología en el sistema 2D, bloque cilindro, con el objetivo posterior de ser aplicada a cualquier sistema 3D, como es el caso de los contactos entre hilos.

Además, se presenta un acelerador de desgaste variable y autoajustable en función del incremento umbral de desgaste y la evolución de la presión de contacto máxima. Todo este proceso se ha desarrollado utilizando la subrutina UMESHMOTION de ABAQUS. que ha sido validada y contrastada por una variedad de autores ${ }^{[9}$ y 11$]$.

\section{METODOLOGÍA EXPERIMENTAL}

\subsection{Ensayos tribológicos}

A la hora de desarrollar la simulación en desgaste, primeramente, se requiere la definición de ensayos tribológicos necesarios, tanto para la obtención del coeficiente de desgaste, que es introducido como input en la simulación, como para la posterior validación de los resultados obtenidos por simulación.

Para este estudio la configuración utilizada fue la de bloque-cilindro (block-on-ring). Las probetas fueron previamente obtenidas por mecanizado de barras de acero C45E. Posteriormente, se les aplicó un tratamiento térmico de temple a $850^{\circ} \mathrm{C}$ y enfriamiento en agua. La dureza obtenida fue de 55-60 HRC. Finalmente, fueron mecanizadas y rectificadas hasta una rugosidad, Ra, de 0,20-0,30 $\mu \mathrm{m}$, medida con un rugosímetro de contacto, para obtener las geometrías y dimensiones deseadas para la realización de los ensayos de las probetas, como se puede apreciar en la figura 2 .

Asimismo, en la tabla I se muestran las dimensiones y rugosidad especificas de cada probeta.

Los ensayos se realizaron en un tribómetro del tipo block-on-ring, en el que se hace girar el disco a una velocidad constante de $0,25 \mathrm{~m} / \mathrm{s}$ y $0,5 \mathrm{~m} / \mathrm{s}$, mientras el bloque es presionado con una fuerza normal de $65 \mathrm{~N}$. Las condiciones del ensayo se muestran especificadas en la tabla II. 


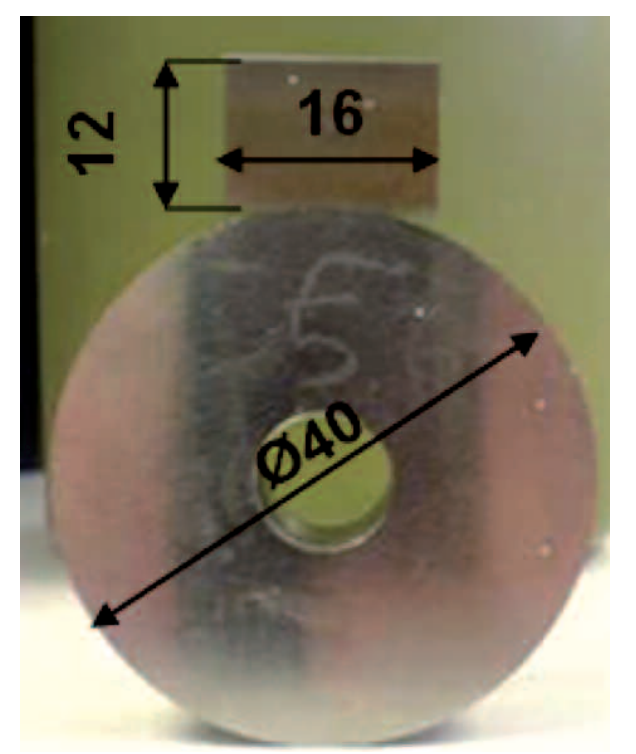

Figura 2. Probetas y sus correspondientes dimensiones.

Figure 2. Testing specimens and the corresponding dimensions.

Tabla I. Dimensiones y rugosidades de las probetas

Table I. Test specimens' dimensions and roughness

\begin{tabular}{lccc}
\hline Probeta & Dimensiones & Rugosidad & $\begin{array}{c}\text { Contact } \\
\text { radius } \\
(\mu \mathrm{m})\end{array}$ \\
\hline Cilindro & $\varnothing 40 \times 11 \mathrm{~mm}$ & $0,22 \mu \mathrm{m}$ & 65 \\
Bloque & $16 \times 12 \times 6 \mathrm{~mm}$ & $0,28 \mu \mathrm{m}$ & 140 \\
\hline
\end{tabular}

\subsection{Medidas tribológicas}

Tras los ensayos realizados, las medidas que se obtienen para poder simular el desgaste son el coeficiente de rozamiento, que se expresa según la fórmula siguiente (Ec. (1)).

$$
\mu=\frac{F_{\mathrm{r}}}{F_{\mathrm{n}}}
$$

donde, $F_{\mathrm{r}}$ se corresponde a la fuerza tangencial de rozamiento y $F_{n}$ a la fuerza normal.

A la hora de la simulación del desgaste se utiliza el coeficiente de rozamiento correspondiente a la segunda
Tabla II. Condiciones de los ensayos tribológicos

Table II. Set of parameters for tribological tests

\begin{tabular}{lcc}
\hline \multicolumn{1}{c}{ Parámetro } & Símbolo & Valor \\
\hline $\begin{array}{l}\text { Fuerza normal }(\mathrm{N}) \\
\text { Presión media inicial }\end{array}$ & $F_{\mathrm{n}}$ & 65 \\
en el contacto (MPa) & $P_{\mathrm{av}}$ & 110 \\
Máxima presión inicial & & \\
en el contacto (MPa) & $P_{\max }$ & 141 \\
Velocidad (m/s) & $v$ & $0,25,0,5$ \\
Tiempo $(\mathrm{h})$ & $t$ & $2,4,5$ \\
Temperatura $\left({ }^{\circ} \mathrm{C}\right)$ & $T$ & $25 \pm 1$ \\
Humedad relativa $(\%)$ & $H R$ & $50 \pm 5$ \\
\hline
\end{tabular}

mitad del ensayo ya que, inicialmente, se observa un periodo en el que el coeficiente de rozamiento varía alcanzando, finalmente, un valor estable ${ }^{[12]}$.

Con respecto al desgaste se obtiene la topografía de las huellas de desgaste por medio de perfilometría confocal, como se puede observar en la figura 3.

A partir de esta topografía se obtienen los perfiles de las huellas de desgaste y el volumen desgastado, el cual permite obtener el coeficiente de desgaste de acuerdo a la siguiente expresión (Ec. (2)).

$$
k=\frac{W_{\mathrm{v}}}{F_{\mathrm{n}} \times s}
$$

donde, $W_{\mathrm{v}}$ se corresponde al volumen desgastado en la pieza, $F_{n}$ corresponde a la fuerza normal aplicada y s corresponde a la distancia total de deslizamiento. De esta manera, para cada ensayo se obtiene un coeficiente de desgaste diferente debido a la dispersión de los resultados experimentales. El coeficiente de desgaste medio se calcularía como la media de estos coeficientes.

Algunos autores ${ }^{[13]}$, proponen otra manera para obtener el coeficiente de desgaste medio de todos los ensayos. Esto es, a partir de la pendiente de la recta obtenida mediante la representación del volumen desgastado para todos los casos estudiados en función del factor de carga de Archard, definido como el producto de la fuerza normal, $F_{n}$, y el deslizamiento total, $s$. Esta manera de obtener el coeficiente de desgaste es la utilizada a la hora de simular el desgaste en este trabajo.

Por otro lado, las topografías de las huellas de desgaste obtenidas, también permiten, tras la simulación, 

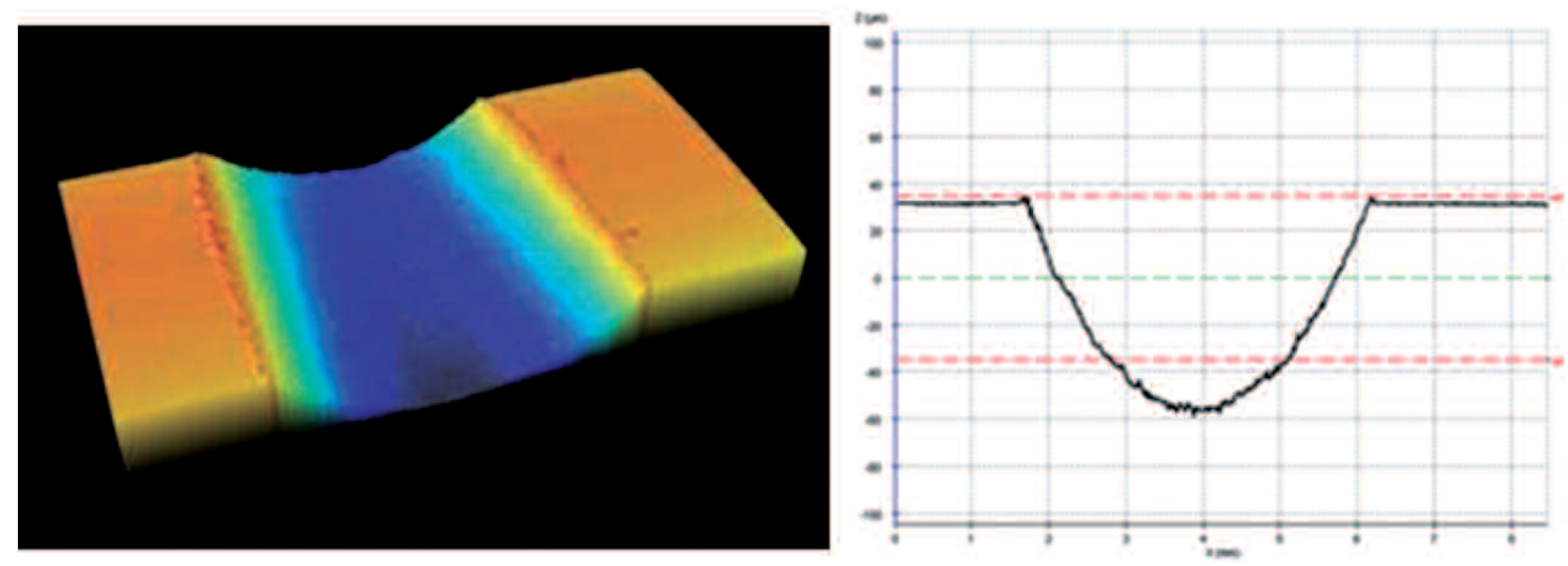

Figura 3. Topografía y perfil de la huella de desgaste en el bloque.

Figure 3. Block's wear scar profile and topography.

la comparación tanto de la anchura de la huella como de la profundidad de esta. De esta forma, se utilizan para validar los resultados obtenidos por simulación.

Hay que señalar que, con el fin de simplificar la simulación y reducir el coste computacional, solo se considera el volumen desgastado en el bloque. Se realiza esta simplificación ya que la profundidad desgastada en el disco (del orden de 0,002 $\mathrm{mm}$ ) es muy pequeña en comparación al radio de éste $(20 \mathrm{~mm})$. Por tanto, se parte de la hipótesis de que el cambio de geometría que se produce como consecuencia del desgaste del disco, apenas influye en la distribución de presiones en el contacto.

\section{METODOLOGIA DE SIMULACIÓN EN DESGASTE}

\subsection{Modelo de contacto MEF}

Se utiliza un modelo bidimensional del sistema blockon-ring, mediante elementos de primer orden de 4 nodos en deformación plana.

La resolución del contacto, teniendo en cuenta el rozamiento, se realiza por medio del método penalti utilizando el algoritmo maestro-esclavo, permitiendo que el maestro penetre en el esclavo. Como en este estudio solo se desgasta el bloque, éste, es considerado como esclavo.

En este estudio hay que tener en cuenta que, con el fin de simplificar el problema y desarrollar la metodología eficientemente se desprecia el deslizamiento en la resolución del contacto. Realizando esta simplificación, se considera que en cada incremento de desgaste todos los puntos deslizan por igual. En casos como este, en el que se produce deslizamiento continuo, esta simplificación resulta eficiente. Sin embargo, si quisiéramos ver el estado tensional debido a la fuerza tangencial o casos en los que el fenómeno de stick-slip tiene gran importancia, esta simplificación no debe considerarse.

\subsection{Modelo de desgaste local}

A la hora de realizar la simulación en desgaste, el segundo concepto a tener en cuenta es el modelo de desgaste utilizado. En este caso, se parte del modelo de Archard (Ec. (2)) donde el coeficiente de desgaste, $k$, se obtiene experimentalmente, como se ha descrito anteriormente.

Si dicho modelo es considerado en cada uno de los puntos de las superficies de contacto, reordenando la ecuación (2) y considerándola por unidad de superficie local se obtiene la expresión de desgaste local (Ec. (3)).

$$
h=k_{1} \times p \times s
$$

En este caso, $h$ representa la profundidad de desgaste local, $p$ la presión de contacto local y $s$ el deslizamiento local. Por otro lado, $k_{1}$ representa el coeficiente de desgaste local. Dicho coeficiente, en principio, no tiene por qué ser el mismo que $k$, obtenido experimentalmente, ya que este coeficiente representa una especie de coeficiente medio considerando toda la huella de desgaste. Sin embargo, estudios realizados por Mccoll et al..$^{[6]}$ han demostrado que tanto el coeficiente de desgaste volumétrico como el local son similares. Debido a la dificultad de poder 
obtener un coeficiente local en todos los casos, se utiliza el coeficiente global.

\subsection{Proceso de simulación en desgaste}

La simulación en desgaste consiste en un proceso incremental que ha de repetirse tantas veces como precisión se necesite. En este caso, la longitud total de deslizamiento se discretiza en tantos incrementos de desgaste como sean necesarios, tal y como se comenta en la introducción. En la figura 4 se puede ver el algoritmo general de desgaste.

Sin embargo, para realizar correcta y óptimamente el proceso anterior, es necesario seguir una metodología optimizada.

De esta manera, en este trabajo se describe una metodología no propuesta anteriormente y que permite realizar la simulación del desgaste de la manera más correcta posible, reduciendo el tiempo computacional, considerablemente. Esta metodología consta de las siguientes etapas:

1. Definición del tamaño de malla en la zona de contacto.

2. Validación del modelo de contacto MEF mediante HERTZ.

3. Encuentro del incremento de desgaste máximo límite para que el sistema no se desestabilice.

4. Simulación del desgaste sin acelerador.

5. Simulación del desgaste mediante acelerador.

6. Comparación del error entre ambos modelos.

7. Validación de la simulación mediante la comparación con perfiles reales.

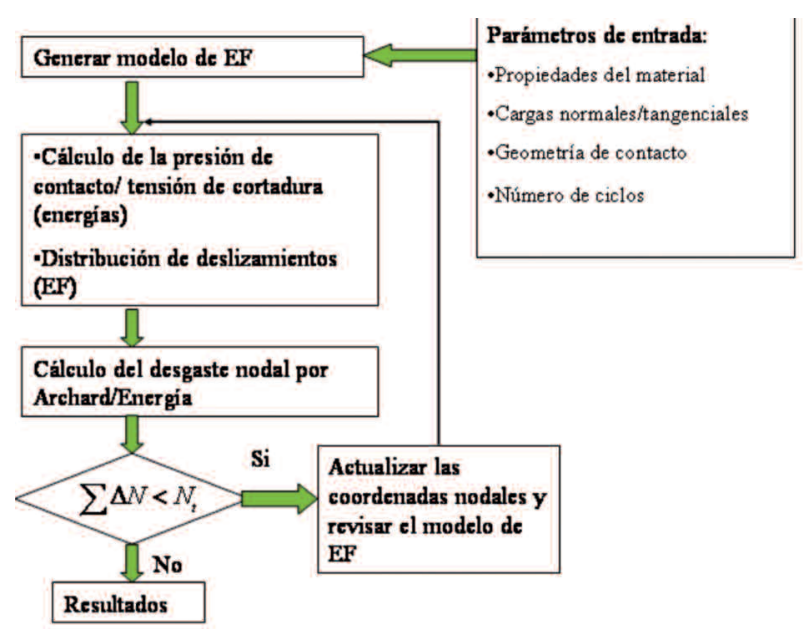

Figura 4. Algoritmo para la simulación del desgaste.

Figure 4. Wear simulation algorithm.

\section{RESULTADOS Y DISCUSIÓN}

El coeficiente de desgaste obtenido en los ensayos bloque-cilindro (block-on-ring) es de $2 \mathrm{E}-09 \mathrm{~mm}^{3} / \mathrm{Nmm}$. En la figura 5 se muestra el modo de obtener este coeficiente de desgaste utilizando el criterio explicado en el apartado 2.2. Por otro lado, el coeficiente de rozamiento obtenido es de 0,65.

Los resultados obtenidos por medio de la simulación numérica se van a estructurar en base a la metodología definida en el apartado 3.3., con el fin de validar cada uno de los pasos seguidos.

En la primera etapa de la metodología propuesta, se realiza la definición del tamaño de malla en la zona de contacto, ya que un tamaño de malla muy fino puede implicar un coste computacional muy alto. Para ello, se realiza un estudio en el que se va a correlacionar el tamaño de malla con la anchura de contacto final, con el fin de obtener el tamaño de malla más grosero posible, sin alterar los resultados finales. Por consiguiente, se consideran dos casos, uno para una distancia de desgaste de $240 \mathrm{~m}$ y otro que simula el ensayo del tribómetro correspondiente a $0,25 \mathrm{~m} / \mathrm{s}$ -2 h (1.800 m).

En la figura 6. se observa que el error cometido en la presión máxima de contacto, a medida de que se aumenta el tamaño de malla, es inferior al $3 \%$. Sin embargo, la parte uniforme de la presión de contacto se reduce considerablemente, p. ej.: en el caso de $240 \mathrm{~m}$ de deslizamiento (Fig. 6 a)), se reduce de 1 a $0,72 \mathrm{~mm}$. Teniendo en cuenta lo anteriormente mencionado, se considera como reducción

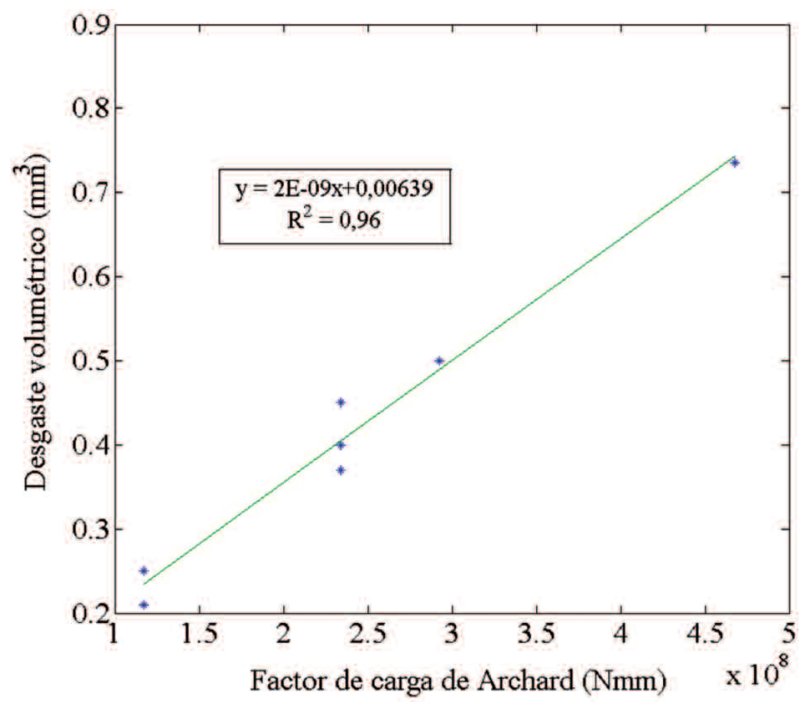

Figura 5. Desgaste volumétrico en función del factor de carga de Archard.

Figure 5. Volumetric wear vs Archard's loading. 


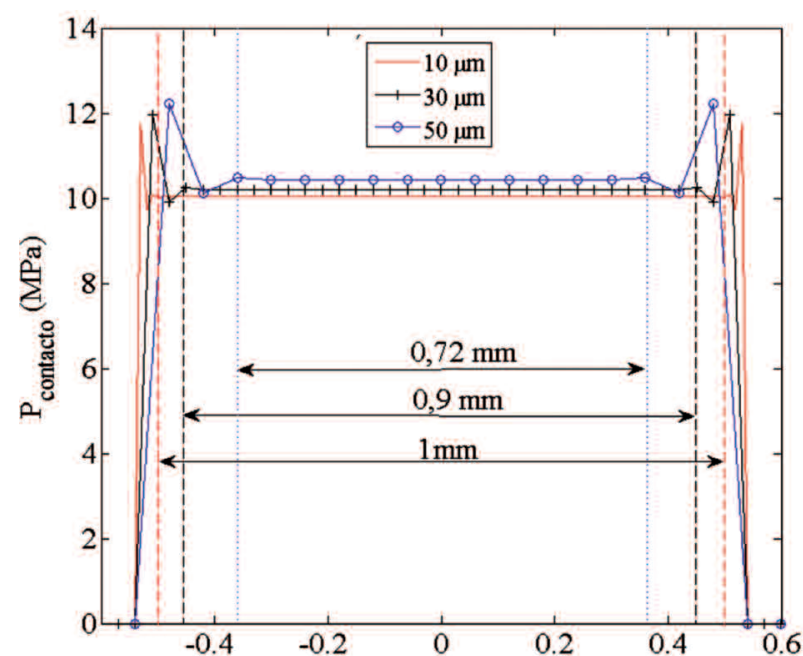

a) Anchura contacto (mm)

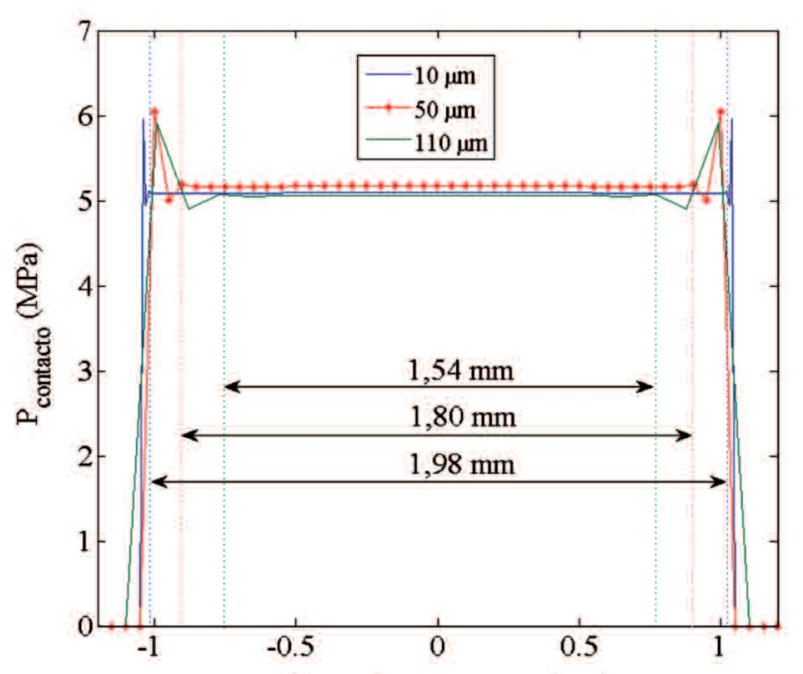

b) Anchura contacto (mm)

Figura 6. Análisis del tamaño de malla con respecto a la presión de contacto: a) distancia de deslizamiento de $240 \mathrm{~m}$; b) distancia de deslizamiento $1.800 \mathrm{~m}$ (ensayo en tribómetro a $0,25 \mathrm{~m} / \mathrm{s}-2 \mathrm{~h})$.

Figure 6. Mesh size analysis with respect to the contact pressure: a) sliding distance $240 \mathrm{~m}$; $b$ ) sliding distance $1,800 \mathrm{~m}(0.25 \mathrm{~m} / \mathrm{s}-2 \mathrm{~h}$ tribometer test).

optima la correspondiente a un $10 \%$ con respecto a la malla más refinada que, para este caso, ha sido la de $10 \mu \mathrm{m}$. Asi pues, mientras para el caso de $240 \mathrm{~m}$ (Fig. 6 a)) la malla optima se encuentra en torno a $30 \mu \mathrm{m}$ para el caso de $1.800 \mathrm{~m}$ (Fig. 6 b)) se encuentra en torno a $50 \mu \mathrm{m}$.

Si se relaciona el tamaño de malla óptima con la anchura de contacto final, en el caso de $240 \mathrm{~m}$, se da una relación de 0,026 y, en el caso de 1800 m, de 0,024 . De este modo, se puede considerar que el tamaño de malla optimo para reducir el tiempo computacional debe estar comprendido entre un 2-3\% de la anchura de contacto final, aproximadamente.

En la segunda etapa, se realiza la validación del modelo MEF mediante las ecuaciones analíticas presentadas por Hertz. En la figura 7 se muestran las diferentes distribuciones de presiones obtenidas en el contacto inicial por las diferentes discretizaciones analizadas anteriormente. Se puede ver, que mientras que las discretizaciones de $5 \mu \mathrm{m}$ y $10 \mu \mathrm{m}$ se ajustan muy bien a Hertz, las de $50 \mu \mathrm{m}$ empiezan a sobredimensionar tanto la anchura de contacto como la presión máxima. Esto implica que, en función del objetivo buscado, será necesario un mayor o menor refinamiento del tamaño de elemento. En el caso de que se quiera analizar la evolución de la presión en las etapas iniciales será necesario un mayor refinamiento del tamaño de elemento. Sin embargo, cuando se quiera analizar el desgaste y presiones finales, tras un número elevado de ciclos, se podrá tomar

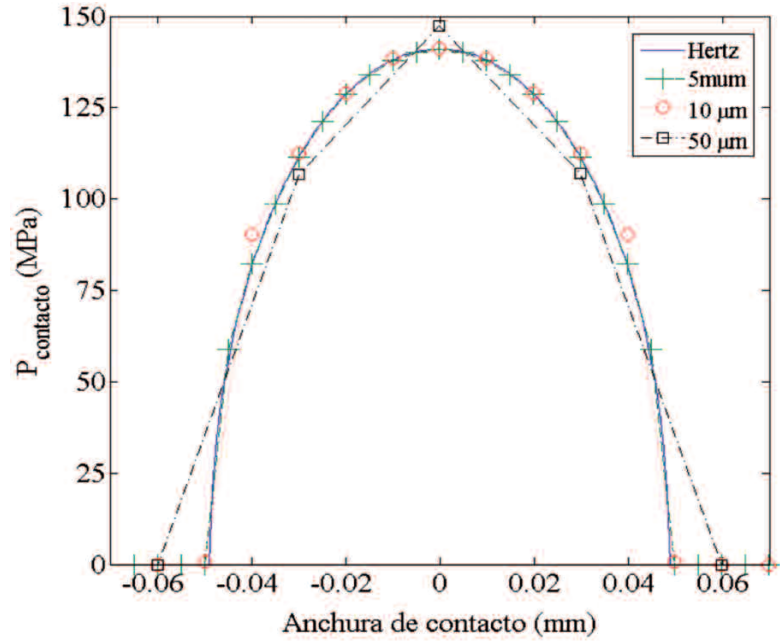

Figura 7. Presión de contacto de Hertz vs. presiones de contacto para las distintas discretizaciones.

Figure 7. Hertz contact pressure vs. contact pressures for different meshes.

un tamaño de elemento más grosero, a pesar de que no se obtenga un buen ajuste de la distribución de Hertz inicial.

En la tercera etapa, se analiza el incremento de desgaste máximo limite para que el sistema no se desestabilice. La ventaja de este estudio es que la inestabilidad 
se ve en los primeros ciclos de desgaste, por lo que el tiempo requerido para encontrar el deslizamiento óptimo no debería resultar demasiado elevado.

En la figura 8 se presenta la distribución de presiones obtenidas para una longitud de deslizamiento total de $480 \mathrm{~mm}$ y diferentes incrementos de deslizamiento, considerándose que durante un incremento de deslizamiento se mantiene constante la distribución de presiones. Esto implica que una mayor discretización aumentará, considerablemente, el tiempo computacional. Se observa que mientras que para 8 incrementos de desgaste aparecen muchas inestabilidades, a medida que se aumenta la discretización, estas inestabilidades van disminuyendo (11 INC). Por otro lado, a partir de 12 incrementos se observa que las presiones de contacto convergen al mismo valor, no observándose ninguna mejora para una discretización más fina (1.000 INC).

Dicho incremento de deslizamiento está directamente relacionado con el incremento máximo de desgaste que se puede dar en cada modelo. En la figura 9 se observa la influencia de la profundidad de desgaste máxima total, tras un ensayo de $480 \mathrm{~mm}$ con respecto al incremento máximo de desgaste. Por un lado, se encuentra el incremento de desgaste máximo umbral. Dicho incremento representa el valor limite a partir del cual el sistema se desestabiliza. Si se disminuye el incremento máximo de desgaste se observa que el error de cálculo disminuye hasta llegar a un valor en el que una discretización más fina no conlleva ninguna mejora en el resultado final. A este valor se le denomina incremento de

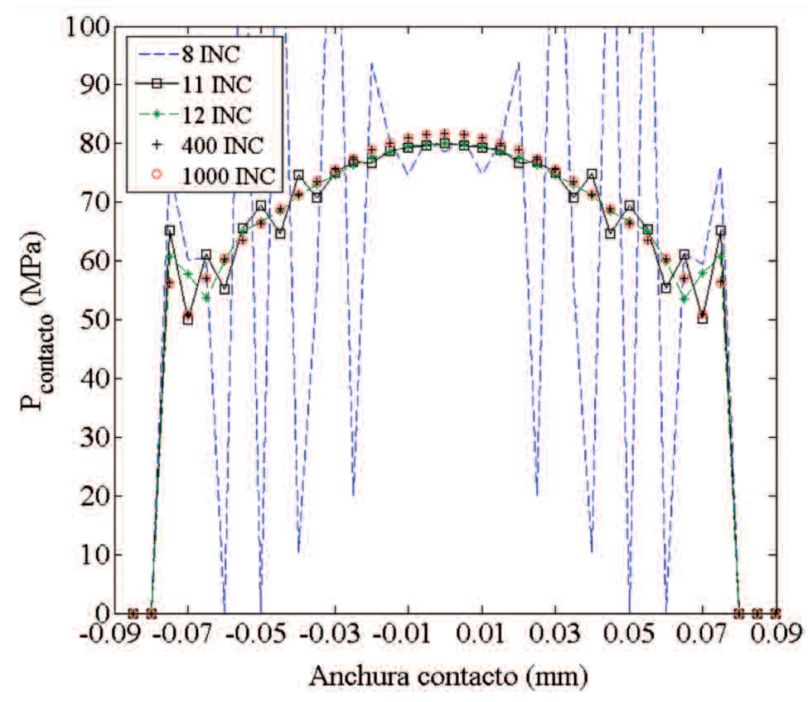

Figura 8. Análisis de la discretización del deslizamiento para una distancia de $480 \mathrm{~mm}$.

Figure 8. Sliding distance discretization analysis for $480 \mathrm{~mm}$.

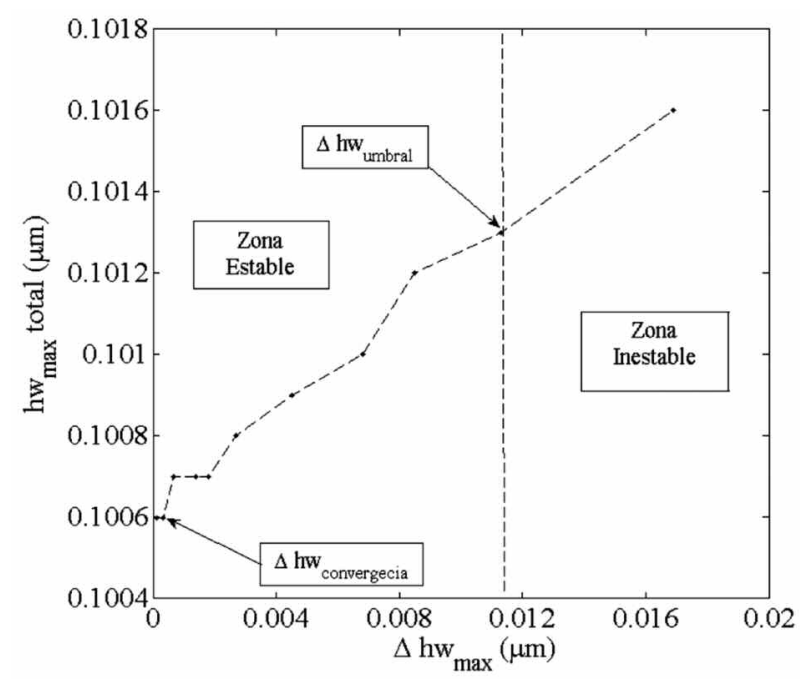

Figura 9. Incremento máximo de desgaste vs. profundidad desgastada para una distancia de $480 \mathrm{~mm}$.

Figure 9. Maximum wear increment vs. wear depth for $480 \mathrm{~mm}$ sliding distance.

desgaste máximo de convergencia. Sin embargo, mientras el error cometido mediante el incremento de desgaste umbral es de 0,5\% la reducción en tiempo computacional es del $95 \%$, por lo que se considera que el valor óptimo es el umbral.

Por tanto, el incremento de desgaste umbral es característico de cada modelo estudiado y depende de la presión de contacto inicial, del coeficiente de desgaste y de la malla utilizada. En este punto hay que remarcar que la malla más grosera posible, tal y como se define en la primera etapa de la metodología propuesta, permite un incremento de desgaste umbral mayor, induciendo una discretización más grosera. Esto implica una reducción de tiempo computacional doble ya que, por un lado, reducimos el número de elementos para el cálculo del contacto y, por otro lado, reducimos el número de veces que se realizan dichos contactos tras aplicar el desgaste.

La cuarta etapa propuesta en esta metodología, es la simulación del desgaste de un ensayo tribológico sin la utilización del acelerador. Este paso sólo se realiza una vez para cada modelo, con el fin de obtener los errores que se puedan cometer una vez introducido el acelerador. En la figura 10 se presenta la distribución de presión máxima en función del deslizamiento total para las tres discretizaciones más groseras presentadas anteriormente. Se observan, claramente, dos periodos: el primero, consiste en el periodo inicial, en el que se observa que la presión de contacto decrece de manera rápida hasta llegar a un segundo periodo, donde la presión de contacto se 


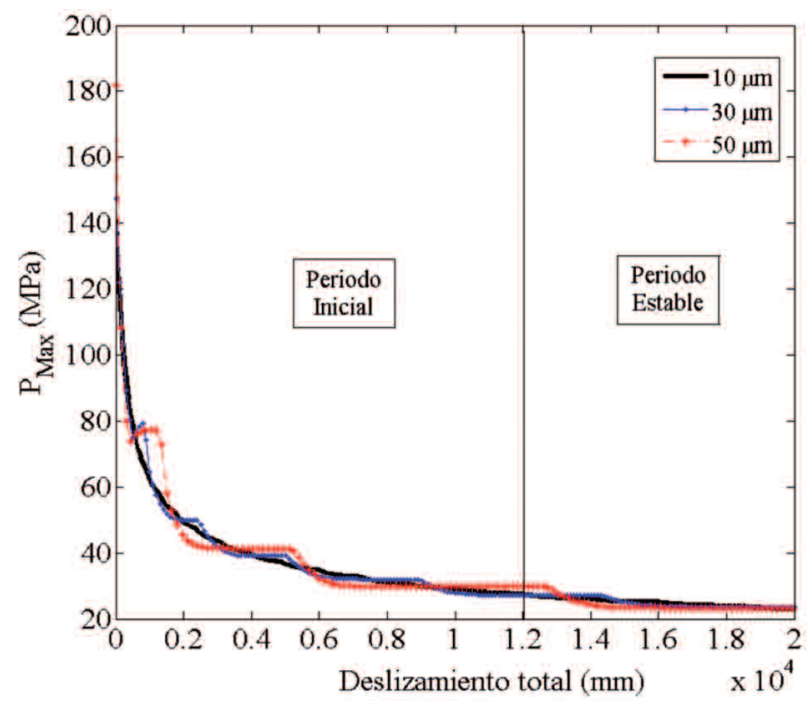

Figura 10. Influencia de la presión máxima de contacto en función del deslizamiento total para el ensayo de $0,25 \mathrm{~m} / \mathrm{s}-2 \mathrm{~h}-65 \mathrm{~N}$ durante los primeros $20 \mathrm{~m}$.

Figure 10. Influence of the maximum contact pressure vs. sliding distance for the test $0.25 \mathrm{~m} / \mathrm{s}-2 \mathrm{~h}-65 \mathrm{~N}$ during de initial $20 \mathrm{~m}$.

mantiene, aproximadamente, constante en toda la anchura de contacto.

Las diferentes discretizaciones únicamente afectan sobre el periodo inicial ya que al inicio del periodo estable, todas ellas, convergen a una misma curva; de esta manera, se observa como la discretización de $50 \mu \mathrm{m}$, definida como óptima para el ensayo de $0,25 \mathrm{~m} / \mathrm{s}-2 \mathrm{~h}$ representa adecuadamente el periodo estable. Así pues, el seguimiento de las etapas anteriores conlleva una gran reducción del tiempo computacional: mientras con una malla de $10 \mu \mathrm{m}$ que se ajusta a Hertz se tardan 18,3 h, con la malla óptima de $50 \mu \mathrm{m}$ se tarda $1,2 \mathrm{~h}$.

La quinta etapa consiste en la introducción del acelerador de desgaste con el fin de reducir considerablemente el tiempo computacional sin afectar al resultado final.

Diversos autores ya han introducido el concepto de utilización de aceleradores de desgaste a lo largo de una simulación completa ${ }^{[14]}$. Sin embargo, en este trabajo se presenta un acelerador de desgaste que presenta dos ventajas respecto a los descritos en la bibliografía, que son especialmente importantes en la simulación de desgastes severos. Por un lado, es un acelerador autoajustable, que consiste en que se define de manera automática el factor de aceleración, tomando como referencia el incremento umbral de desgaste. Por otro lado, la introducción del acelerador se realiza automáticamente en función del diferencial de presiones que se considere. Este criterio de introducción del acelerador tiene como objetivo aplicarlo en el momento que se llega al periodo estable. No se introduce en el periodo inicial, con el objetivo de modelizar adecuadamente la fuerte variación de presiones que se produce en este periodo. Hay que indicar que, en todos los casos, se deja de aplicar, de manera automática, durante los últimos 300 ciclos, con el fin de eliminar todas las inestabilidades producidas. En la figura 11 se aprecia este efecto, de modo que, una vez eliminado el acelerador, la presión de contacto converge a la obtenida en la simulación sin la aplicación del acelerador.

En la sexta etapa se realiza el estudio del error obtenido, tanto en profundidad de desgaste como en anchura de contacto, mediante la introducción del acelerador de desgaste. En ambos casos, se obtienen errores inferiores al $1 \%$, consiguiendo una reducción del tiempo del $87 \%$.

La última etapa consiste en correlacionar los resultados obtenidos experimentalmente con los obtenidos mediante simulación, utilizando el modelo optimizado de desgaste. La figura 12 muestra la correlación entre la geometría obtenida experimentalmente y la obtenida por simulación mediante MEF. Teniendo en cuenta que en ensayos tribológicos se estiman dispersiones de hasta el $15 \%$, podría decirse que los resultados obtenidos mediante simulación se ajustan a los obtenidos experiementalmente.

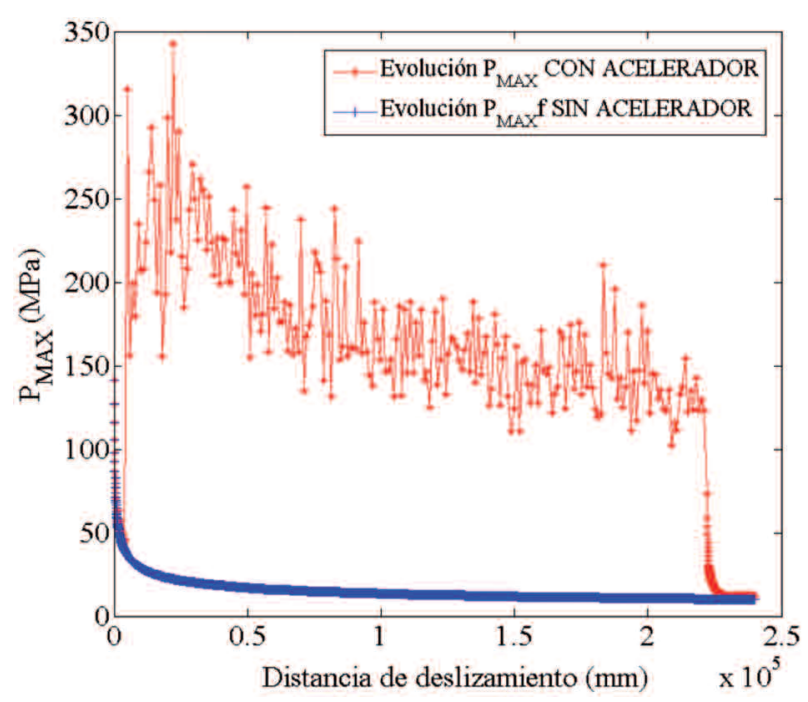

Figura 11. Efecto de la introducción del acelerador de desgaste en la presión máxima de contacto.

Figure 11. The wear accelerator effect in the maximum contact pressure. 


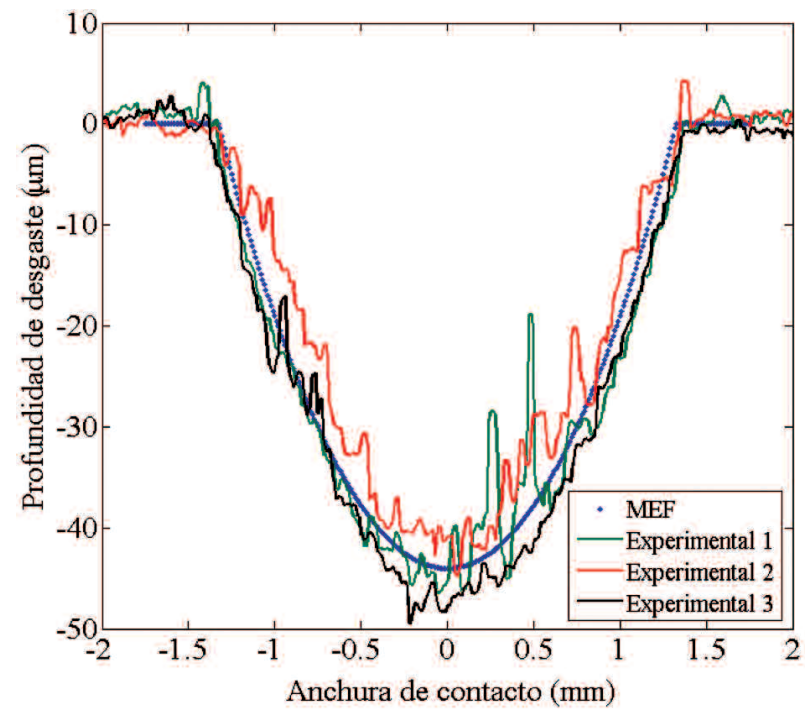

Figura 12. Huella de desgaste experimental vs. MEF para el ensayo de $0,25 \mathrm{~m} / \mathrm{s}-2 \mathrm{~h}-65 \mathrm{~N}$.

\section{Figure 12. Experimental wear scar vs. FEM for} the test $0.25 \mathrm{~m} / \mathrm{s}-2 \mathrm{~h}-65 \mathrm{~N}$.

Finalmente, se puede observar la gran reducción de tiempo computacional que se puede conseguir mediante la utilización de esta metodología. Ya que mientras que utilizando una malla de $10 \mu \mathrm{m}$ se tarda un tiempo de $18 \mathrm{~h}$ con una malla de $50 \mu \mathrm{m}$ con acelerador se reduce a $12 \mathrm{~min}$.

\section{CONCLUSIONES}

En este trabajo se ha desarrollado una metodología eficiente para realizar la simulación del desgaste mediante la optimización del tiempo computacional. Primeramente, se ha analizado la influencia del tamaño de la malla en la zona de contacto, considerándose tamaño de malla óptimo, el comprendido entre un 2-3\% de la anchura de contacto final. Por otro lado, se ha demostrado la correlación que hay entre el tamaño de malla utilizado y la discretización de incremento de desgaste: un mayor tamaño de elemento permite la reducción del número de incrementos de desgaste. Esta metodología permite la reducción de un modelo refinado que se ajusta a Hertz, el cual necesita 18,3 h, a un modelo óptimo, que solo necesita $1,2 \mathrm{~h}$.

Además, se ha introducido un acelerador de desgate, el cual entra automáticamente durante la zona estable y se autoajusta a una profundidad de desgaste límite, que el usuario define. La mejora computacional es de un $87 \%$ aproximado, en tiempo, con un error inferior al $1 \%$ en geometría.

Finalmente, se ha obtenido una buena correlación entre los resultados experimentales y el modelo optimizado presentado en este trabajo. Asi pues, se presenta como una herramienta muy util para las simulaciones de desgaste de materiales metálicos que requieran un alto coste computacional, como por ejemplo: en desgastes severos (en los cuales son necesarias muchas iteraciones de desgaste) y en sistemas 3D.

\section{Agradecimientos}

Los autores quieren agradecer al departamento de Educación, Universidades e Investigación del Gobierno Vasco la financiación concedida al proyecto SIVICA (Ref. UE2009-5) a través del programa Universidad-Empresa.

\section{REFERENCIAS}

[1] M.A. Urchegui, W. Tato y X. Gómez, J. Mater. Eng. Perform. 17 (2007) 550-560.

[2] P. Podra y S. Andersson, Tribol. Int. 32 (1999) 71-81.

[3] V. Hegadekatte, N. Huber y O. Kraft, Modelling. Simul. Mater. Sci. Eng. 13 (2005) 57-75.

[4] N.H. Kim, D. Won, D. Burris, B. Holtkamp, G.R. Gessel, P. Swanson y W.G. Sawyer, Wear 25 (2005) 1.787-1.793.

[5] M. Oqvist, Wear 249 (2001) 6-11.

[6] I.R. McColl, J. Ding y S.B. Leen, Wear 256 (2004) 1.114-1.1127.

[7] S. Fouvry, C. Paulin y C. Meunier, Wear 264 (2008) 26-36.

[8] J. Ding, I.R. McColl y S.B. Leen, Wear 262 (2007) 1.205-1.216.

[9] R. Hernández, M. Riera y J.M. Prado, Reunión de usuarios de Abaqus, Madrid, 2006, http:// www.principia.es/abaqus/usuarios_archivo.htm (obtenida 25.02.2011).

[10] V. Hegadekatte, S. Kurzenhäuser, N. Huber, O. Kraft, Tribol. Int. 41 (2008) 1.020-1.031.

[11] J. Ding, S.B. Leen, E.J. Willians y P.H. Shipway, Tribol. Mater. Surf. Interf. 2 (2008) 10-24.

[12] A. Cruzado, M. Hartelt, R. Wäsche, M.A. Urchegui y X. Gómez, Wear 268 (2010) 1.409. 1.416.

[13] S. Fouvry, T. Liskiewicz y C. Paulin, Wear 263 (2007) 518-531.

[14] J. Ding, Thesis, University of Nothingan, 2008. 